\title{
LIMING AND IONIC SPECIATION OF AN OXISOL UNDER NO-TILL SYSTEM
}

\author{
Fernando Cesar Bachiega Zambrosi ${ }^{1 *}$; Luís Reynaldo Ferracciú Alleoni², Eduardo Fávero \\ Caires $^{3}$ \\ ${ }^{1}$ IAC - Centro de Pesquisa e Desenvolvimento de Solos e Recursos Ambientais, Av. Barão de Itapura, 1481 - \\ 13001-970 - Campinas, SP - Brasil. \\ ${ }_{3}^{2}$ USP/ESALQ - Depto. de Ciência do Solo, C.P. 09 - 13418-900 - Piracicaba, SP - Brasil. \\ ${ }^{3}$ UEPG - Depto. de Ciência do Solo e Engenharia Agrícola, Av. Carlos Cavalcanti, 4748 - 84030-900 - Ponta \\ Grossa, PR - Brasil. \\ *Corresponding author 〈zambrosi@iac.sp.gov.br>
}

\begin{abstract}
The chemical speciation of the soil solution is an important tool for evaluating nutrient availability and aluminum phytotoxicity. The effect of liming in the composition of the soil solution under the no-till (NT) system is still not well known. We evaluated the chemical speciation of $\mathrm{Al}$ and nutrients in the soil solution (water extract 1:1), five years after liming at the moment of the establishment of NT in a native pasture, in Ponta Grossa, Paraná State, Brazil. Dolomitic lime was applied at a rate of $4.5 \mathrm{t} \mathrm{ha}^{-1}$ (either incorporated or surface-applied), or split in three annual rates of $1.5 \mathrm{t} \mathrm{ha}^{-1}$. Soil samples from a dystrophic clayey Rhodic Hapludox were collected at the $0-0.05 ; 0.05-0.1 ; 0.1-0.2 ; 0.2-0.4 ; 0.4-0.6$; and 0.6 - 0.8 m layers, in May, 2003. Aluminum was mainly complexed to dissolved organic carbon (DOC) and fluoride, while the free form $\mathrm{Al}^{3+}$ was present in fewer amounts even under acidic conditions. Calcium and magnesium were found as free forms $\left(\mathrm{Ca}^{2+}, \mathrm{Mg}^{2+}\right)$ and complexed by organic anions. Phosphorus was found as free forms $\left(\mathrm{HPO}_{4}^{2-}, \mathrm{H}_{2} \mathrm{PO}_{4}^{-}\right)$and complexed with aluminum (ionic pair $\left.\mathrm{Al}-\mathrm{H}_{\mathrm{x}} \mathrm{PO}_{4}^{\mathrm{x}}\right)$ up to $0.1 \mathrm{~m}$. Complexation of $\mathrm{Al}$ by DOC was not influenced by surface-applied or incorporated liming. Liming did not change $\mathrm{Ca}$ and $\mathrm{Mg}$ speciation in the soil solution under NT system. The presence of free forms $\mathrm{HPO}_{4}^{2-}$ and $\mathrm{H}_{2} \mathrm{PO}_{4}^{-}$, however, was influenced by liming, because of the increase in cation associated forms.

Key words: dolomitic lime, complex, ionic pair, dissolved organic carbon
\end{abstract}

\section{CALAGEME ESPECIAÇÃO IÔNICA DE UM LATOSSOLO SOB SISTEMA PLANTIO DIRETO}

RESUMO: A especiação química da solução do solo é uma importante ferramenta para avaliar a disponibilidade de nutrientes e o potencial fitotóxico do alumínio. As formas em que os elementos ocorrem na solução dos solos sob sistema plantio direto (SPD) ainda são pouco conhecidas, sobretudo em áreas que receberam aplicação de calcário. Avaliou-se a distribuição das espécies químicas de alumínio e nutrientes na solução do solo (extrato aquoso 1:1), cinco anos após a aplicação de calcário, na introdução do SPD em área de pastagem nativa, em Ponta Grossa -PR. Aplicaram-se 4,5 $\mathrm{t} \mathrm{ha}^{-1}$ de calcário dolomítico na superfície, em dose única ou parcelada durante três anos, ou com incorporação na camada de 0 - 0,20 $\mathrm{m}$, de um Latossolo Vermelho distrófico textura argilosa. As amostras de solo foram coletadas nas profundidades de 0 - 0,05; 0,05 - 0,1;0,1 - 0,2;0,2 - 0,4;0,4 - 0,6 e 0,6 - 0,8 m. O alumínio (Al) ocorreu predominantemente complexado pelo carbono orgânico dissolvido (DOC) e pelo fluoreto, e em baixíssimas proporções na forma livre $\mathrm{Al}^{3+}$, mesmo em condições de alta acidez. Para cálcio $(\mathrm{Ca})$ e magnésio $(\mathrm{Mg})$, as

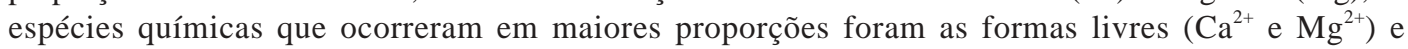
complexadas pelo DOC. O fósforo, além das formas livres $\left(\mathrm{H}_{2} \mathrm{PO}_{4}^{-}, \mathrm{HPO}_{4}^{2-}\right)$, interagiu com alumínio (par iônico $\mathrm{Al}-\mathrm{H}_{\mathrm{x}} \mathrm{PO}_{4}{ }^{\mathrm{x}}$ ), sobretudo até $0,1 \mathrm{~m}$ de profundidade. A complexação do alumínio pelo carbono orgânico dissolvido não foi influenciada pela correção da acidez do solo por meio da calagem na superfície ou com incorporação. A aplicação de calcário não alterou a especiação de cálcio e magnésio na solução do solo sob sistema plantio direto. Contudo, a ocorrência das formas livres $\mathrm{H}_{2} \mathrm{PO}_{4}^{-}$e $\mathrm{HPO}_{4}^{2-}$ foi influenciada pela calagem, havendo incremento das formas associadas com cátions.

Palavras-chave: calcário dolomítico, complexos, par iônico, carbono orgânico dissolvido

\section{INTRODUCTION}

The no-till (NT) system is an efficient soil con- servation practice, nevertheless, there is a lack of information on soil fertility and crop management for this system (Rheinheimer et al., 2000). The better ero- 
sion control as compared to the conventional tillage system leads to smaller losses of organic carbon and nutrients, mainly $\mathrm{Ca}$ and $\mathrm{Mg}$ (Bertol et al., 2005). Consequently, the area under NT in Brazil has grown in the last few years. Regarding soil fertility management, there has been an increase in studies about liming in NT, focusing on forms and rates of application and correction of soil acidity (Alleoni et al., 2003, 2005; Amaral \& Anghinoni, 2001; Caires et al., 1998, 1999, 2000, 2004; Kaminski et al., 2005). Assessments on the effects of liming on soil chemical attributes should be made in a long term basis in order to obtain the residual effects of liming (Caires et al., 2005, 2006b). Nevertheless, studies that also focus on the soil solution under NT, especially through chemical speciation, are scarce. In a recent study with samples of an Oxisol collected in areas of distinct acidity conditions, and later placed in columns with several rates of phosphorus, Nolla \& Anghinoni (2006) pointed out the importance of chemical speciation in the evaluation of the phytotoxic potential of aluminum in soils cultivated under the NT system.

The main soil chemical reactions happen in soil solution. Therefore, knowledge of its composition is very important, both for environmental management studies and for soil fertility and plant nutrition studies. The soil-solution interface is very dynamic, and the dissolved elements are affected by several reactions, such as acid-base equilibrium, ionic complexation, precipitation and dissolution of solids, oxidation, reduction and ionic exchange (Chaves et al., 1991; Fontes \& Alleoni, 2006). The concentration of most dissolved cations in the solution is determined by the equilibrium with their exchangeable forms (Nemeth et al., 1970). Liming in acid soils is, most likely, the agricultural practice with the greatest potential for changing the composition of the exchange complex and the liquid phase, due to the quick equilibrium between phases. Thus, liming affects the dynamics of dissolved ions (Amaral et al., 1998; Zambrosi et al., 2007).

A detailed study of the soil solution thorough chemical speciation contributes to a better understanding of the mobility and availability of nutrients or phytotoxic metals (Krishnamurtiand \& Naidu, 2002), despite the variability in the methods of obtaining and the dependence on edafoclimatic conditions (Wolt, 1994). In the soil solution, most of the ions are present either as free ions or interacting with other ions or molecules, forming soluble complexes (Sposito, 1994). In areas under the NT system, the presence of relatively high concentrations of organic ligands in the soil solution, due to the increment in organic matter, can reduce aluminum toxicity (Hue et al., 1986) and help mobilize cations (Tack \& Verloo, 1995). When com- pared to the soil total organic matter, the more readily available fraction, such as the dissolved organic carbon (DOC), is more sensitive to changes caused by soil management in the short and medium terms, being, therefore, used as a fundamental indicator of soil quality or of changes in native conditions (Silveira, 2005).

Chemical speciation provides a clear view of the interactions of elements in soil solution. Such interaction interferes in nutrient mobility and availability, and can help elucidate the mechanisms involved in acidity amelioration by liming in soils under NT. In the present study, the percentage of aluminum and nutrient chemical species in the soil solution of an Oxisol under NT was quantified, five years after liming, surface applied or incorporated, with single and split applications.

\section{MATERIAL AND METHODS}

Samples from a dystrophic clayey Rhodic Hapludox were collected in a field experiment set up in 1998, in a native pasture area located in Ponta Grossa, Paraná State, Brazil $\left(25^{\circ} 10^{\prime} \mathrm{S}, 50^{\circ} 05^{\prime} \mathrm{W}\right)$. The soil chemical analyses performed before the beginning of the experiment, following the methodology described by Pavan et al. (1992), indicated the following results, for the $0-0.2$ and $0.2-0.4 \mathrm{~m}$ soil layers, respectively: $\mathrm{pH} \mathrm{CaCl}{ }_{2}$ of 4.6 and 4.2 ; organic matter (OM) content of 31 and $21 \mathrm{~g} \mathrm{dm}^{-3}$; P (Mehlich-1) of 0.3 and $0.1 \mathrm{mg} \mathrm{dm}^{-3} ; \mathrm{K}^{+}$of 3.6 and $2.2 ; \mathrm{Ca}^{2+}$ of 25 and $7 ; \mathrm{Mg}^{2+}$ of 20 and $8 ; \mathrm{Al}^{3+}$ of 3 and $8 ; \mathrm{H}+\mathrm{Al}$ of 77.6 and $97.0 \mathrm{mmol}_{\mathrm{c}} \mathrm{dm}^{-3}$; base saturation $(\mathrm{V})$ of 38 and $15 \%$; aluminum saturation (m) of 6 and $32 \%$. The soil textural composition, determined according to Embrapa (1997) methodology for these same soil layers, was 290 and $300 \mathrm{~g} \mathrm{~kg}^{-1}$ sand; 130 and $100 \mathrm{~g} \mathrm{~kg}^{-1}$ silt; 580 and $600 \mathrm{~g} \mathrm{~kg}^{-1}$ clay, respectively.

The experiment was carried out in a completely randomized block design with three replications. Dolomitic lime with an effective calcium carbonate equivalent (ECCE) of $89 \%$ was used at a rate calculated to raise the soil base saturation to $70 \%$ in the 0 $0.2 \mathrm{~m}$ layer. The treatments were: control (no lime) (T1); surface application of $4.5 \mathrm{t} \mathrm{ha}^{-1}$ split in three annual applications of $1.5 \mathrm{t} \mathrm{ha}^{-1}$ (T2); a single surface application of $4.5 \mathrm{t} \mathrm{ha}^{-1}$ (T3); a single application of $4.5 \mathrm{t} \mathrm{ha}^{-1}$ incorporated to $0.2 \mathrm{~m}$ (T4). Lime was applied by hand to the soil surface in July 1998. In the incorporated lime plots, one-half of the lime was surface broadcasted by hand, and incorporated to a 0.2 $m$ depth with a disk plow. The remainder was added by hand to the surface, and the soil was harrowed to a $0.1 \mathrm{~m}$ depth with a disk harrow. In the plots with 
annual splitting of surface-applied lime, the second application was performed in May 1999, and the third, in May 2000.

The sequence of crops was soybean (19981999), barley (winter of 1999), soybean (1999-2000), wheat (winter of 2000), soybean (2000-2001), corn (2001-2002), and soybean (2002-2003). For the soybean crop, the fertilizer application at seeding was 75 $\mathrm{kg} \mathrm{ha}^{-1}$ of $\mathrm{P}_{2} \mathrm{O}_{5}$ and $\mathrm{K}_{2} \mathrm{O}\left(300 \mathrm{~kg} \mathrm{ha}^{-1}\right.$ of the $0-25-25$ formula) in the first crop, and $60 \mathrm{~kg} \mathrm{ha}^{-1}$ of $\mathrm{P}_{2} \mathrm{O}_{5}$ and $\mathrm{K}_{2} \mathrm{O}$ (300 kg ha ${ }^{-1}$ of the 0-20-20 formula) in the three other crops. The fertilizers used for the soybeans contained $5 \% \mathrm{~S}$, as $\mathrm{CaSO}_{4}$, from the simple superphosphate. Thus, each soybean crop received $15 \mathrm{~kg} \mathrm{ha}^{-1}$ of $\mathrm{S}$. The barley crop received $28 \mathrm{~kg} \mathrm{ha}^{-1}$ of $\mathrm{N}$ and 92 $\mathrm{kg} \mathrm{ha}^{-1}$ of $\mathrm{P}_{2} \mathrm{O}_{5}$, as diammonium phosphate (DAP) at sowing, and $45 \mathrm{~kg} \mathrm{ha}^{-1}$ of $\mathrm{N}$, as top-dressed urea. The wheat crop received $33 \mathrm{~kg} \mathrm{ha}^{-1}$ of $\mathrm{N}$ and $66 \mathrm{~kg} \mathrm{ha}^{-1}$ of $\mathrm{P}_{2} \mathrm{O}_{5}$ and $\mathrm{K}_{2} \mathrm{O}$ (330 kg ha-1 of the 10-20-20 formula) at sowing, and $100 \mathrm{~kg} \mathrm{ha}^{-1}$ of $\mathrm{N}$, as top-dressed urea. Corn crop fertilization was $413 \mathrm{~kg} \mathrm{ha}^{-1}$ of the 8-30-20 formula at sowing, and top-dressing of $124 \mathrm{~kg} \mathrm{ha}^{-1}$ of the 36-00-12 formula. Insect, weed and disease management was performed as needed, according to each crops' recommendations. The cropping sequence for each liming treatment was described by Caires et al. (2006a).

Soil samples were collected in May, 2003, right after the soybean harvest, five years after liming. Samples were taken with a probe, collecting 12 subsamples per plot, forming a composite sample for the $0-0.05,0.05-0.1$, and $0.1-0.2 \mathrm{~m}$ layers, and five subsamples per plot for the $0.2-0.4,0.4-0.6$, and 0.6 - $0.8 \mathrm{~m}$ layers. Air-dried samples were gently crushed and passed through a $2 \mathrm{~mm}$ sieve, stored in plastic bags and kept in the shade at room temperature until analysis. The samples were analyzed for $\mathrm{pH}$, $\mathrm{Al}^{3+}, \mathrm{Ca}^{2+}, \mathrm{Mg}^{2+}, \mathrm{K}^{+}$, and total organic carbon, according to Pavan et al. (1992).

The water-extraction method (Wolt, 1994) was employed extract the soil solution, using a soil:water ratio of $1: 1$. In a $50 \mathrm{~mL}$ centrifuge tube, $20 \mathrm{~mL}$ of ultrapure water were added to $20 \mathrm{~g}$ of soil. Then, the tubes were placed in a horizontal shaker for $15 \mathrm{~min}$ utes at $150 \mathrm{rpm}$, and left resting for $1 \mathrm{~h}$. After this period, the solution was shaken again for five more minutes (Wolt, 1994) and centrifuged for 30 minutes at $1500 \mathrm{rpm}$. Three extractions were performed to obtain cation, anion and dissolved organic carbon (DOC) extracts. To obtain the extract for cation determination, the solution was passed through a $0.45 \mathrm{~mm}$ mesh cellulose membrane. Another extract was passed through a $0.22 \mathrm{~mm}$ mesh cellulose membrane for anion determination. The extract for DOC was obtained by passing the solution through a $0.7 \mathrm{~mm}$ mesh $\mathrm{GF} / \mathrm{F}$ glass microfiber filter, previously calcinated at $500^{\circ} \mathrm{C}$ for $6 \mathrm{~h}$, and stored in flasks submitted to the same calcination process.

The filtering processes to obtain the extracts were performed with a $13 \mathrm{~mm}$ diameter filter support for the cation and anion fractions, and a $25 \mathrm{~mm}$ diameter filter support for the DOC. These supports were connected to $60 \mathrm{~mL}$ plastic syringes, where the extracts were placed after centrifugation, and were filtered by manual pressure. The $\mathrm{pH}$ was determined in the solutions immediately after centrifugation. The total contents of calcium, magnesium, potassium, sodium, aluminum, iron, and manganese were determined by ICP-OES, while nitrate, sulfate, phosphate, chloride, and fluoride contents were determined using a chromatographer. The DOC was determined in a Shimadzu 5000A carbon analyzer.

The soil solution ionic strength (I) was calculated as follows:

$I=1 / 2 \sum_{i=1}^{n} C i \cdot(Z i)^{2}$

where $\mathrm{Ci}$ is the concentration $\left(\mathrm{mol} \mathrm{L}^{-1}\right)$, and $\mathrm{Zi}$ is the charge of each ion $i$ in solution.

The ionic speciation of the soil solution was performed with the Visual MINTEQ computer program (Gustafsson, 2004), using the total concentration (mg $\mathrm{L}^{-1}$ ) of cations, inorganic anions, and DOC, and the ionic strength $\left(\mathrm{mol} \mathrm{L}^{-1}\right)$ and $\mathrm{pH}$ values of the solution. The distribution in percentages of each species in the soil solution was obtained as a function of the forms of limestone application and sampling depths. The percentages of each species were compared within each depth, using the mean standard error (with three replications).

\section{RESULTS AND DISCUSSION}

Lime application reduced the exchangeable aluminum content and increased the exchangeable calcium and magnesium contents and soil $\mathrm{pH}$, especially at the $0-0.2$ layer (Table 1). The composition of soil solution (water extract) are presented in Tables 2 and 3. In general, liming increased soil $\mathrm{pH}$ and soluble calcium and magnesium contents, especially in the surface layers. Down to the $0.1-0.2 \mathrm{~m}$ depth, there was a positive $(P<0.05)$ correlation between the exchangeable and soluble content values of calcium and magnesium (data not shown), confirming the equilibrium between these two phases, as well as the sensitivity of the water extract in reflecting changes in the exchangeable content of these nutrients in the soil. The correlation between calcium and magnesium in solu- 
Table 1 - Lime treatments, contents of exchangeable of cations, organic carbon (C), and values of $\mathrm{pH}$ for different depths of an Oxisol under no-till system. Values between parenthesis represent the mean deviation $(n=3)$.

\begin{tabular}{|c|c|c|c|c|c|c|}
\hline \multirow[t]{2}{*}{ Treat* } & $\mathrm{Ca}$ & $\mathrm{Mg}$ & K & $\mathrm{Al}$ & $\mathrm{pH}$ & $\mathrm{C}$ \\
\hline & \multicolumn{5}{|c|}{$\begin{array}{l}-1 \\
-\end{array}$} & $\mathrm{g} \mathrm{kg}^{-1}$ \\
\hline & \multicolumn{6}{|c|}{$0-0.05 \mathrm{~m}$} \\
\hline $\mathrm{T} 1$ & $37.7 \pm(1.9)$ & $24.3 \pm(3.8)$ & $6.4 \pm(0.7)$ & $1.0 \pm(0.0)$ & $4.9 \pm(0.0)$ & $33.3 \pm(1.3)$ \\
\hline $\mathrm{T} 2$ & $59.7 \pm(0.9)$ & $46.0 \pm(1.5)$ & $5.3 \pm(0.7)$ & $0.0 \pm(0.0)$ & $5.9 \pm(0.0)$ & $31.7 \pm(1.5)$ \\
\hline $\mathrm{T} 3$ & $63.0 \pm(2.1)$ & $42.7 \pm(1.3)$ & $5.3 \pm(0.2)$ & $0.0 \pm(0.0)$ & $5.8 \pm(0.1)$ & $35.0 \pm(1.5)$ \\
\hline \multirow[t]{2}{*}{$\mathrm{T} 4$} & $50.7 \pm(4.8)$ & $36.7 \pm(5.2)$ & $6.7 \pm(0.7)$ & $0.0 \pm(0.0)$ & $5.5 \pm(0.1)$ & $31.3 \pm(1.5)$ \\
\hline & \multicolumn{6}{|c|}{$0.05-0.1 \mathrm{~m}$} \\
\hline $\mathrm{T} 1$ & $25.7 \pm(0.9)$ & $17.0 \pm(4.0)$ & $4.6 \pm(0.7)$ & $3.3 \pm(0.9)$ & $4.6 \pm(0.0)$ & $27.0 \pm(2.1)$ \\
\hline $\mathrm{T} 2$ & $33.7 \pm(0.9)$ & $27.0 \pm(1.0)$ & $3.1 \pm(0.5)$ & $0.0 \pm(0.0)$ & $5.1 \pm(0.1)$ & $31.3 \pm(5.4)$ \\
\hline $\mathrm{T} 3$ & $32.7 \pm(3.2)$ & $26.0 \pm(0.6)$ & $3.1 \pm(0.3)$ & $0.0 \pm(0.0)$ & $5.3 \pm(0.1)$ & $24.7 \pm(1.2)$ \\
\hline \multirow[t]{2}{*}{$\mathrm{T} 4$} & $40.7 \pm(1.8)$ & $34.7 \pm(4.1)$ & $5.0 \pm(0.9)$ & $0.0 \pm(0.0)$ & $5.5 \pm(0.1)$ & $26.7 \pm(1.9)$ \\
\hline & \multicolumn{6}{|c|}{$0.1-0.2 \mathrm{~m}$} \\
\hline $\mathrm{T} 1$ & $15.0 \pm(1.0)$ & $15.7 \pm(2.0)$ & $2.7 \pm(0.6)$ & $4.3 \pm(0.9)$ & $4.6 \pm(0.1)$ & $21.0 \pm(1.2)$ \\
\hline $\mathrm{T} 2$ & $18.3 \pm(1.5)$ & $15.7 \pm(2.0)$ & $1.6 \pm(0.3)$ & $3.0 \pm(0.0)$ & $4.8 \pm(0.0)$ & $21.0 \pm(2.0)$ \\
\hline $\mathrm{T} 3$ & $16.3 \pm(1.8)$ & $14.3 \pm(3.3)$ & $1.7 \pm(0.2)$ & $3.0 \pm(0.6)$ & $4.8 \pm(0.0)$ & $18.7 \pm(0.9)$ \\
\hline \multirow[t]{2}{*}{$\mathrm{T} 4$} & $25.3 \pm(2.4)$ & $24.0 \pm(1.0)$ & $2.9 \pm(0.2)$ & $0.0 \pm(0.0)$ & $5.2 \pm(0.1)$ & $22.3 \pm(2.3)$ \\
\hline & \multicolumn{6}{|c|}{$0.2-0.4 \mathrm{~m}$} \\
\hline $\mathrm{T} 1$ & $13.7 \pm(1.8)$ & $14.7 \pm(2.7)$ & $2.3 \pm(1.1)$ & $4.7 \pm(0.9)$ & $4.6 \pm(0.1)$ & $19.0 \pm(1.0)$ \\
\hline $\mathrm{T} 2$ & $17.3 \pm(2.7)$ & $14.3 \pm(0.7)$ & $1.8 \pm(0.3)$ & $3.0 \pm(1.0)$ & $4.5 \pm(0.1)$ & $18.7 \pm(1.8)$ \\
\hline $\mathrm{T} 3$ & $15.3 \pm(2.7)$ & $15.7 \pm(3.3)$ & $1.3 \pm(0.1)$ & $2.3 \pm(0.9)$ & $4.5 \pm(0.0)$ & $18.7 \pm(1.5)$ \\
\hline \multirow[t]{2}{*}{$\mathrm{T} 4$} & $15.0 \pm(1.5)$ & $18.7 \pm(0.9)$ & $1.7 \pm(0.1)$ & $0.7 \pm(0.3)$ & $4.7 \pm(0.0)$ & $18.7 \pm(0.9)$ \\
\hline & \multicolumn{6}{|c|}{$0.4-0.6 \mathrm{~m}$} \\
\hline $\mathrm{T} 1$ & $8.0 \pm(1.0)$ & $10.0 \pm(2.1)$ & $1.1 \pm(0.5)$ & $4.7 \pm(0.3)$ & $4.5 \pm(0.0)$ & $14.0 \pm(1.2)$ \\
\hline $\mathrm{T} 2$ & $11.0 \pm(0.6)$ & $14.3 \pm(1.9)$ & $1.0 \pm(0.3)$ & $2.0 \pm(0.0)$ & $4.8 \pm(0.0)$ & $13.7 \pm(1.8)$ \\
\hline $\mathrm{T} 3$ & $9.0 \pm(0.6)$ & $9.3 \pm(0.3)$ & $0.6 \pm(0.1)$ & $1.7 \pm(0.3)$ & $4.8 \pm(0.1)$ & $13.7 \pm(1.3)$ \\
\hline \multirow[t]{2}{*}{$\mathrm{T} 4$} & $8.3 \pm(1.2)$ & $11.0 \pm(1.2)$ & $2.1 \pm(1.1)$ & $2.7 \pm(0.3)$ & $4.7 \pm(0.0)$ & $13.7 \pm(1.5)$ \\
\hline & \multicolumn{6}{|c|}{$0.6-0.8 \mathrm{~m}$} \\
\hline $\mathrm{T} 1$ & $7.0 \pm(1.0)$ & $10.7 \pm(2.2)$ & $1.2 \pm(0.8)$ & $3.0 \pm(0.6)$ & $4.7 \pm(0.0)$ & $11.7 \pm(0.7)$ \\
\hline $\mathrm{T} 2$ & $8.0 \pm(0.6)$ & $10.0 \pm(1.2)$ & $0.5 \pm(0.1)$ & $2.3 \pm(0.3)$ & $4.8 \pm(0.0)$ & $11.7 \pm(0.7)$ \\
\hline $\mathrm{T} 3$ & $10.7 \pm(2.3)$ & $8.7 \pm(0.3)$ & $1.2 \pm(0.7)$ & $2.3 \pm(0.7)$ & $4.8 \pm(0.1)$ & $16.3 \pm(4.5)$ \\
\hline $\mathrm{T} 4$ & $7.0 \pm(0.6)$ & $9.7 \pm(1.2)$ & $1.9 \pm(0.9)$ & $2.7 \pm(0.7)$ & $4.7 \pm(0.1)$ & $10.3 \pm(0.7)$ \\
\hline
\end{tabular}

*T1 $=$ control (without lime); $\mathrm{T} 2=$ three annual surface liming of $1.5 \mathrm{tha}^{-1} ; \mathrm{T} 3=4.5 \mathrm{t} \mathrm{ha}^{-1}$ of surface-applied lime; $\mathrm{T} 4=4.5 \mathrm{t} \mathrm{ha}^{-1}$ of lime incorporated at $0.2 \mathrm{~m}$ depth.

tion and their exchangeable content was also observed by Ciotta et al. (2004) in an Oxisol under NT after surface and incorporated application of lime.

Aluminum was detected in free form $\left(\mathrm{Al}^{3+}\right)$ and, in greater proportion, forming complexes in the soil solution (Figure 1). Plant toxicity decreases in the following order: $\mathrm{Al}_{13}$ polymers (except for phosphates and silicates), $\mathrm{Al}^{3+}, \mathrm{Al}(\mathrm{OH})^{2+}, \mathrm{Al}(\mathrm{OH})_{2}^{+}, \mathrm{Al}(\mathrm{OH})_{4}^{-}$, and $\mathrm{AlSO}_{4}^{+}$, considering that the toxicity of this last species is not always accepted (Drabeck et al., 2005). The Al species bonded with fluoride and organic compounds, $\mathrm{Al}(\mathrm{OH})_{3}$, are considered nontoxic. Although
$\mathrm{Al}_{13}$ is the most toxic species, it is not commonly present in soils (Drabeck et al., 2005). The free form $\mathrm{Al}^{3+}$ is, therefore, the most harmful to plant roots. Nevertheless, in the present study, there were proportionally low concentrations of $\mathrm{Al}^{3+}$, regardless of the depth sampled, even in the treatment without liming. Under natural conditions, low or moderate concentrations of $\mathrm{Al}^{3+}$ are usually observed in the solution of acid soils, while the complexes formed between aluminum and fluoride (Al-F) and aluminum and dissolved organic carbon (Al-DOC) represent the majority of $\mathrm{Al}$ species (Merino et al., 1998). 
Table 2 - Lime treatments, values of $\mathrm{pH}$, and total concentration of dissolved organic carbon (DOC) and inorganic anions in solution for different depths of an Oxisol under no-till system. Values between parenthesis represent the mean deviation $(\mathrm{n}=3)$.

\begin{tabular}{|c|c|c|c|c|c|c|c|}
\hline Treat.* & $\mathrm{pH}$ & DOC & $\mathrm{N}-\mathrm{NO}_{3}^{-}$ & $\mathrm{Cl}$ & $\mathrm{S}-\mathrm{SO}_{4}{ }^{2-}$ & $\mathrm{P}-\mathrm{PO}_{4}{ }^{3-}$ & $\mathrm{F}$ \\
\hline & & & & 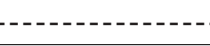 & $\mathrm{mmol} \mathrm{L}^{-1}-$ & & \\
\hline & & & & $0-0$. & $5 \mathrm{~m}$ & & \\
\hline $\mathrm{T} 1$ & $5.0 \pm(0.0)$ & $16.8 \pm(0.4)$ & $0.27 \pm(0.01)$ & $0.16 \pm(0.04)$ & $0.057 \pm(0.004)$ & $0.0011 \pm(0.0009)$ & $0.11 \pm(0.00)$ \\
\hline $\mathrm{T} 2$ & $6.2 \pm(0.1)$ & $15.0 \pm(0.3)$ & $0.24 \pm(0.00)$ & $0.24 \pm(0.07)$ & $0.080 \pm(0.006)$ & $0.0010 \pm(0.0005)$ & $0.18 \pm(0.03)$ \\
\hline $\mathrm{T} 3$ & $5.9 \pm(0.0)$ & $17.7 \pm(1.0)$ & $0.29 \pm(0.02)$ & $0.16 \pm(0.01)$ & $0.073 \pm(0.022)$ & $0.0002 \pm(0.0000)$ & $0.14 \pm(0.06)$ \\
\hline \multirow[t]{2}{*}{$\mathrm{T} 4$} & $5.5 \pm(0.2)$ & $18.4 \pm(1.2)$ & $0.30 \pm(0.02)$ & $0.17 \pm(0.03)$ & $0.055 \pm(0.001)$ & $0.0001 \pm(0.0001)$ & $0.11 \pm(0.05)$ \\
\hline & \multicolumn{7}{|c|}{$0.05-0.1 \mathrm{~m}$} \\
\hline $\mathrm{T} 1$ & $4.8 \pm(0.1)$ & $16.5 \pm(2.5)$ & $0.27 \pm(0.04)$ & $0.18 \pm(0.04)$ & $0.026 \pm(0.003)$ & $0.0014 \pm(0.0009)$ & $0.08 \pm(0.02)$ \\
\hline $\mathrm{T} 2$ & $5.5 \pm(0.2)$ & $14.8 \pm(0.0)$ & $0.24 \pm(0.00)$ & $0.10 \pm(0.02)$ & $0.040 \pm(0.008)$ & $0.0001 \pm(0.0000)$ & $0.05 \pm(0.02)$ \\
\hline T3 & $5.1 \pm(0.1)$ & $16.2 \pm(1.6)$ & $0.26 \pm(0.03)$ & $0.13 \pm(0.05)$ & $0.048 \pm(0.017)$ & $0.0002 \pm(0.0001)$ & $0.08 \pm(0.03)$ \\
\hline \multirow[t]{2}{*}{$\mathrm{T} 4$} & $5.3 \pm(0.1)$ & $15.5 \pm(1.5)$ & $0.25 \pm(0.02)$ & $0.09 \pm(0.02)$ & $0.021 \pm(0.006)$ & $0.0002 \pm(0.0000)$ & $0.03 \pm(0.00)$ \\
\hline & \multicolumn{7}{|c|}{$0.1-0.2 \mathrm{~m}$} \\
\hline $\mathrm{T} 1$ & $4.8 \pm(0.1)$ & $12.1 \pm(1.9)$ & $0.20 \pm(0.03)$ & $0.15 \pm(0.03)$ & $0.015 \pm(0.003)$ & $0.0001 \pm(0.0000)$ & $0.07 \pm(0.01)$ \\
\hline $\mathrm{T} 2$ & $5.0 \pm(0.1)$ & $10.7 \pm(0.5)$ & $0.17 \pm(0.01)$ & $0.21 \pm(0.06)$ & $0.023 \pm(0.002)$ & $0.0003 \pm(0.0001)$ & $0.06 \pm(0.01)$ \\
\hline $\mathrm{T} 3$ & $4.9 \pm(0.2)$ & $11.7 \pm(1.2)$ & $0.19 \pm(0.02)$ & $0.12 \pm(0.00)$ & $0.018 \pm(0.002)$ & $0.0004 \pm(0.0002)$ & $0.06 \pm(0.02)$ \\
\hline \multirow[t]{2}{*}{$\mathrm{T} 4$} & $5.2 \pm(0.0)$ & $10.8 \pm(0.3)$ & $0.17 \pm(0.01)$ & $0.20 \pm(0.06)$ & $0.031 \pm(0.007)$ & $0.0001 \pm(0.0000)$ & $0.07 \pm(0.01)$ \\
\hline & \multicolumn{7}{|c|}{$0.2-0.4 \mathrm{~m}$} \\
\hline $\mathrm{T} 1$ & $4.9 \pm(0.1)$ & $6.4 \pm(1.0)$ & $0.10 \pm(0.02)$ & $0.27 \pm(0.10)$ & $0.010 \pm(0.002)$ & $0.0002 \pm(0.0001)$ & $0.04 \pm(0.01)$ \\
\hline $\mathrm{T} 2$ & $5.0 \pm(0.1)$ & $6.0 \pm(0.4)$ & $0.10 \pm(0.01)$ & $0.14 \pm(0.01)$ & $0.018 \pm(0.009)$ & $0.0001 \pm(0.0001)$ & $0.04 \pm(0.00)$ \\
\hline T3 & $4.8 \pm(0.2)$ & $6.9 \pm(1.0)$ & $0.11 \pm(0.02)$ & $0.17 \pm(0.04)$ & $0.010 \pm(0.004)$ & $0.0002 \pm(0.0002)$ & $0.04 \pm(0.01)$ \\
\hline \multirow[t]{2}{*}{ T4 } & $4.9 \pm(0.1)$ & $6.8 \pm(0.2)$ & $0.11 \pm(0.00)$ & $0.22 \pm(0.11)$ & $0.023 \pm(0.004)$ & $\mathrm{nd}^{* *}$ & $0.05 \pm(0.01)$ \\
\hline & \multicolumn{7}{|c|}{$0.4-0.6 \mathrm{~m}$} \\
\hline $\mathrm{T} 1$ & $4.8 \pm(0.1)$ & $4.6 \pm(0.7)$ & $0.07 \pm(0.01)$ & $0.27 \pm(0.03)$ & $0.001 \pm(0.001)$ & $0.0001 \pm(0.0001)$ & $0.03 \pm(0.00)$ \\
\hline $\mathrm{T} 2$ & $5.0 \pm(0.0)$ & $3.6 \pm(0.1)$ & $0.06 \pm(0.00)$ & $0.31 \pm(0.06)$ & $0.011 \pm(0.009)$ & $0.0003 \pm(0.0002)$ & $0.03 \pm(0.00)$ \\
\hline $\mathrm{T} 3$ & $4.7 \pm(0.2)$ & $4.0 \pm(0.7)$ & $0.06 \pm(0.01)$ & $0.30 \pm(0.04)$ & $0.001 \pm(0.000)$ & $0.0002 \pm(0.0002)$ & $0.03 \pm(0.00)$ \\
\hline \multirow[t]{2}{*}{ T4 } & $4.7 \pm(0.2)$ & $3.7 \pm(0.4)$ & $0.06 \pm(0.01)$ & $0.19 \pm(0.05)$ & $0.001 \pm(0.000)$ & $0.0001 \pm(0.0001)$ & $0.03 \pm(0.01)$ \\
\hline & \multicolumn{7}{|c|}{$0.6-0.8 \mathrm{~m}$} \\
\hline $\mathrm{T} 1$ & $4.8 \pm(0.1)$ & $2.6 \pm(0.4)$ & $0.04 \pm(0.01)$ & $0.30 \pm(0.04)$ & $0.011 \pm(0.002)$ & $0.0001 \pm(0.0001)$ & $0.02 \pm(0.00)$ \\
\hline $\mathrm{T} 2$ & $4.9 \pm(0.2)$ & $2.4 \pm(0.1)$ & $0.04 \pm(0.00)$ & $0.30 \pm(0.02)$ & $0.001 \pm(0.001)$ & $0.0002 \pm(0.0001)$ & $0.01 \pm(0.00)$ \\
\hline $\mathrm{T} 3$ & $4.8 \pm(0.1)$ & $2.5 \pm(0.1)$ & $0.04 \pm(0.00)$ & $0.22 \pm(0.09)$ & $0.002 \pm(0.000)$ & $0.0001 \pm(0.0000)$ & $0.02 \pm(0.00)$ \\
\hline T4 & $4.7 \pm(0.2)$ & $2.8 \pm(0.2)$ & $0.04 \pm(0.00)$ & $0.23 \pm(0.05)$ & $0.001 \pm(0.000)$ & nd & $0.01 \pm(0.00)$ \\
\hline
\end{tabular}

$* \mathrm{~T} 1=$ control (without lime); $\mathrm{T} 2=4.5 \mathrm{t} \mathrm{ha}^{-1}$ of surface-applied lime (split in three annual applications of $1.5 \mathrm{tha} \mathrm{h}^{-1}$ ); $\mathrm{T} 3=4.5 \mathrm{t} \mathrm{ha}{ }^{-1}$ of surface-applied lime (single application); $\mathrm{T} 4=4.5 \mathrm{t} \mathrm{ha}^{-1}$ of lime incorporated at $0.2 \mathrm{~m}$ depth.**nd: not detected.

In the NT system, organic anions have an important role in $\mathrm{Al}$ chemistry, being the main responsible for reduction of the activity of this element in solution, and thus, reducing its phytoxicity (Vance et al., 1996). The complexation of Al by DOC, in general, was not affected by the liming treatments. Cambri et al. (2004) observed that the proportion of Al-DOC in the soil solution under NT was approximately $85 \%$ in the $0-0.2 \mathrm{~m}$ layer, and that this proportion was little affected by surface application of lime. Adams et al. (1999) verified that the Al bonded to DOC was strongly correlated to the concentration of dissolved humic acid substances in acid soils of New Zealand.

Fluoride (F), which competed intensely with DOC, was another ligand responsible for the strong complexation of $\mathrm{Al}$ in the soil solution (Figure 1). While DOC and fluoride complexed aluminum in a large scale, other anions such as sulfate $\left(\mathrm{S}^{-\mathrm{SO}_{4}^{2-}}\right)$ and phosphate $\left(\mathrm{P}-\mathrm{H}_{\mathrm{x}} \mathrm{PO}_{4}{ }^{\mathrm{x}}\right)$ formed ionic pairs in a much smaller proportion, representing, at the most, $0.3 \%$ of the total Al species (other species). Sulfate and 
Table 3 - Lime treatments, total concentration of cations, and ionic strength (I) of solution for different depths of an Oxisol under no-till system. Values between parenthesis represent the mean deviation $(\mathrm{n}=3)$.

\begin{tabular}{|c|c|c|c|c|c|c|c|c|}
\hline Treat.* & $\mathrm{Al}$ & $\mathrm{Ca}$ & $\mathrm{Mg}$ & $\mathrm{K}$ & $\mathrm{Na}$ & $\mathrm{Fe}$ & $\mathrm{Mn}$ & $\mathrm{I}$ \\
\hline & - & & $-z_{1}$ & $-\cdots \mathrm{mr}$ & $\mathrm{mol} \mathrm{L} \mathrm{L}^{-1}-$ & -- & $\cdots$ & \\
\hline & & & & $0-$ & $0.05 \mathrm{~m}$ & & & \\
\hline $\mathrm{T} 1$ & $0.25 \pm(0.01)$ & $0.32 \pm(0.02)$ & $0.36 \pm(0.03)$ & $0.82 \pm(0.13)$ & $0.06 \pm(0.01)$ & $0.084 \pm(0.062)$ & $0.003 \pm(0.000)$ & $4.6 \pm(1.0)$ \\
\hline $\mathrm{T} 2$ & $0.29 \pm(0.08)$ & $0.55 \pm(0.02)$ & $0.80 \pm(0.02)$ & $0.66 \pm(0.11)$ & $0.06 \pm(0.02)$ & $0.031 \pm(0.008)$ & $0.001 \pm(0.000)$ & $5.6 \pm(0.3)$ \\
\hline $\mathrm{T} 3$ & $0.25 \pm(0.06)$ & $0.55 \pm(0.05)$ & $0.78 \pm(0.06)$ & $0.68 \pm(0.04)$ & $0.06 \pm(0.02)$ & $0.029 \pm(0.011)$ & $0.001 \pm(0.000)$ & $5.2 \pm(0.6)$ \\
\hline \multirow[t]{2}{*}{$\mathrm{T} 4$} & $0.98 \pm(0.44)$ & $0.38 \pm(0.02)$ & $0.54 \pm(0.04)$ & $0.68 \pm(0.08)$ & $0.06 \pm(0.01)$ & $0.090 \pm(0.066)$ & $0.001 \pm(0.000)$ & $7.9 \pm(2.2)$ \\
\hline & \multicolumn{8}{|c|}{$0.05-0.1 \mathrm{~m}$} \\
\hline $\mathrm{T} 1$ & $0.40 \pm(0.14)$ & $0.19 \pm(0.00)$ & $0.18 \pm(0.01)$ & $0.43 \pm(0.06)$ & $0.06 \pm(0.02)$ & $0.032 \pm(0.011)$ & $0.001 \pm(0.000)$ & $3.4 \pm(0.7)$ \\
\hline $\mathrm{T} 2$ & $0.38 \pm(0.03)$ & $0.23 \pm(0.03)$ & $0.37 \pm(0.04)$ & $0.28 \pm(0.06)$ & $0.05 \pm(0.02)$ & $0.025 \pm(0.003)$ & $0.001 \pm(0.000)$ & $3.7 \pm(0.4)$ \\
\hline $\mathrm{T} 3$ & $0.42 \pm(0.09)$ & $0.23 \pm(0.02)$ & $0.37 \pm(0.02)$ & $0.34 \pm(0.02)$ & $0.05 \pm(0.03)$ & $0.037 \pm(0.01)$ & $0.001 \pm(0.000)$ & $4.1 \pm(0.6)$ \\
\hline \multirow[t]{2}{*}{$\mathrm{T} 4$} & $0.65 \pm(0.24)$ & $0.31 \pm(0.01)$ & $0.45 \pm(0.02)$ & $0.36 \pm(0.04)$ & $0.04 \pm(0.01)$ & $0.048 \pm(0.020)$ & $0.001 \pm(0.000)$ & $5.7 \pm(1.6)$ \\
\hline & \multicolumn{8}{|c|}{$0.1-0.2 \mathrm{~m}$} \\
\hline $\mathrm{T} 1$ & $0.09 \pm(0.05)$ & $0.13 \pm(0.02)$ & $0.16 \pm(0.01)$ & $0.29 \pm(0.03)$ & $0.05 \pm(0.02)$ & $0.010 \pm(0.006)$ & $0.001 \pm(0.000)$ & $1.6 \pm(0.2)$ \\
\hline $\mathrm{T} 2$ & $0.10 \pm(0.04)$ & $0.16 \pm(0.01)$ & $0.26 \pm(0.01)$ & $0.21 \pm(0.03)$ & $0.05 \pm(0.02)$ & $0.013 \pm(0.006)$ & $0.001 \pm(0.000)$ & $1.9 \pm(0.2)$ \\
\hline $\mathrm{T} 3$ & $0.07 \pm(0.02)$ & $0.14 \pm(0.00)$ & $0.22 \pm(0.01)$ & $0.20 \pm(0.02)$ & $0.05 \pm(0.02)$ & $0.009 \pm(0.003)$ & $0.001 \pm(0.000)$ & $1.6 \pm(0.1)$ \\
\hline \multirow[t]{2}{*}{$\mathrm{T} 4$} & $0.53 \pm(0.31)$ & $0.21 \pm(0.02)$ & $0.36 \pm(0.01)$ & $0.22 \pm(0.03)$ & $0.04 \pm(0.01)$ & $0.025 \pm(0.004)$ & $0.001 \pm(0.000)$ & $4.2 \pm(1.4)$ \\
\hline & \multicolumn{8}{|c|}{$0.2-0.4 \mathrm{~m}$} \\
\hline $\mathrm{T} 1$ & $0.05 \pm(0.05)$ & $0.12 \pm(0.00)$ & $0.15 \pm(0.01)$ & $0.22 \pm(0.06)$ & $0.06 \pm(0.03)$ & $0.007 \pm(0.007)$ & $0.001 \pm(0.000)$ & $1.4 \pm(0.3)$ \\
\hline $\mathrm{T} 2$ & $0.01 \pm(0.00)$ & $0.14 \pm(0.01)$ & $0.21 \pm(0.02)$ & $0.15 \pm(0.02)$ & $0.05 \pm(0.01)$ & $0.001 \pm(0.000)$ & $0.001 \pm(0.000)$ & $1.2 \pm(0.1)$ \\
\hline $\mathrm{T} 3$ & $0.01 \pm(0.00)$ & $0.14 \pm(0.02)$ & $0.22 \pm(0.01)$ & $0.11 \pm(0.01)$ & $0.03 \pm(0.01)$ & nd** & $0.001 \pm(0.000)$ & $1.2 \pm(0.1)$ \\
\hline \multirow[t]{2}{*}{$\mathrm{T} 4$} & $0.10 \pm(0.09)$ & $0.14 \pm(0.02)$ & $0.27 \pm(0.04)$ & $0.12 \pm(0.03)$ & $0.03 \pm(0.01)$ & $0.001 \pm(0.000)$ & $0.001 \pm(0.000)$ & $1.8 \pm(0.5)$ \\
\hline & \multicolumn{8}{|c|}{$0.4-0.6 \mathrm{~m}$} \\
\hline $\mathrm{T} 1$ & $0.01 \pm(0.00)$ & $0.08 \pm(0.01)$ & $0.12 \pm(0.02)$ & $0.10 \pm(0.04)$ & $0.04 \pm(0.02)$ & nd & $0.003 \pm(0.000)$ & $0.8 \pm(0.1)$ \\
\hline $\mathrm{T} 2$ & $0.02 \pm(0.01)$ & $0.10 \pm(0.02)$ & $0.14 \pm(0.03)$ & $0.06 \pm(0.00)$ & $0.03 \pm(0.01)$ & $0.007 \pm(0.006)$ & $0.008 \pm(0.006)$ & $1.0 \pm(0.2)$ \\
\hline $\mathrm{T} 3$ & $0.01 \pm(0.00)$ & $0.09 \pm(0.00)$ & $0.12 \pm(0.01)$ & $0.05 \pm(0.02)$ & $0.03 \pm(0.01)$ & nd & $0.002 \pm(0.000)$ & $0.8 \pm(0.0)$ \\
\hline \multirow[t]{2}{*}{$\mathrm{T} 4$} & $0.01 \pm(0.00)$ & $0.08 \pm(0.02)$ & $0.13 \pm(0.02)$ & $0.05 \pm(0.02)$ & $0.03 \pm(0.01)$ & nd & $0.003 \pm(0.000)$ & $0.7 \pm(0.2)$ \\
\hline & \multicolumn{8}{|c|}{$0.6-0.8 \mathrm{~m}$} \\
\hline $\mathrm{T} 1$ & $0.01 \pm(0.00)$ & $0.09 \pm(0.01)$ & $0.10 \pm(0.01)$ & $0.07 \pm(0.03)$ & $0.05 \pm(0.02)$ & nd & $0.004 \pm(0.000)$ & $0.8 \pm(0.1)$ \\
\hline $\mathrm{T} 2$ & $0.01 \pm(0.00)$ & $0.08 \pm(0.02)$ & $0.12 \pm(0.02)$ & $0.04 \pm(0.01)$ & $0.07 \pm(0.03)$ & $0.001 \pm(0.000)$ & $0.004 \pm(0.001)$ & $0.8 \pm(0.1)$ \\
\hline T3 & $0.07 \pm(0.07)$ & $0.09 \pm(0.02)$ & $0.14 \pm(0.05)$ & $0.04 \pm(0.01)$ & $0.05 \pm(0.03)$ & $0.028 \pm(0.028)$ & $0.027 \pm(0.023)$ & $1.2 \pm(0.7)$ \\
\hline $\mathrm{T} 4$ & $0.01 \pm(0.01)$ & $0.06 \pm(0.01)$ & $0.11 \pm(0.00)$ & $0.03 \pm(0.02)$ & $0.02 \pm(0.00)$ & $0.001 \pm(0.000)$ & $0.005 \pm(0.000)$ & $0.6 \pm(0.0)$ \\
\hline
\end{tabular}

phosphate have similar values of ligand constant to $\mathrm{Al}$, both much smaller than the values of ligand constant of fluoride and Al (Lindsay \& Walthall, 1996). In many instances, the chemical species $\mathrm{Al}-\mathrm{H}_{\mathrm{x}} \mathrm{PO}_{4}{ }^{\mathrm{x}}$ $\left(\mathrm{AlHPO}_{4}^{+}+\mathrm{Al}_{2} \mathrm{PO}_{4}^{3+}\right)$ was practically not detected, due to the very low concentration of phosphate in the soil solution (Table 2). For the conditions of the present study, both sulfate and phosphate had little influence on Al behavior. Álvarez et al. (2002) reported that the $\mathrm{AlSO}_{4}^{+}$species rarely occurred in Spanish soils with different parent materials. Nolla \& Anghinoni (2006) verified that aluminum phosphate was not present in the soil solution of an Oxisol under NT, even after the application of high rates of $\mathrm{P}$.
Regardless of the treatment or the sampling depth, calcium speciation was basically divided into the free form $\left(\mathrm{Ca}^{2+}\right)$ and the complexation of $\mathrm{Ca}$ with the dissolved OM fraction in the soil solution (Ca-DOC) (Figure 2). The Ca-DOC was predominant in the soil solution, except for the $0-0.05$ and $0.05-0.1$ $\mathrm{m}$-layers, which reflects the marked influence of DOC in the speciation of $\mathrm{Ca}$ in soil solution. Sulfate was the inorganic anion which formed more ionic pairs with $\mathrm{Ca}\left(\mathrm{CaSO}_{4}{ }^{0}\right)$, but always with values under $1 \%$. There were also associations of calcium with fluoride $\left(\mathrm{CaF}^{+}\right)$, phosphate $\left(\mathrm{CaHPO}_{4(\mathrm{aqq}} ; \mathrm{CaH}_{2} \mathrm{PO}_{4}^{+}\right)$, chloride $\left(\mathrm{CaCl}^{+}\right)$, and nitrate $\left(\mathrm{CaNO}_{3}^{+}\right)$, designated as "other species" in Figure 2, representing, when added up, less than $1 \%$ of the total $\mathrm{Ca}$ species. The levels of association of $\mathrm{Ca}$ 

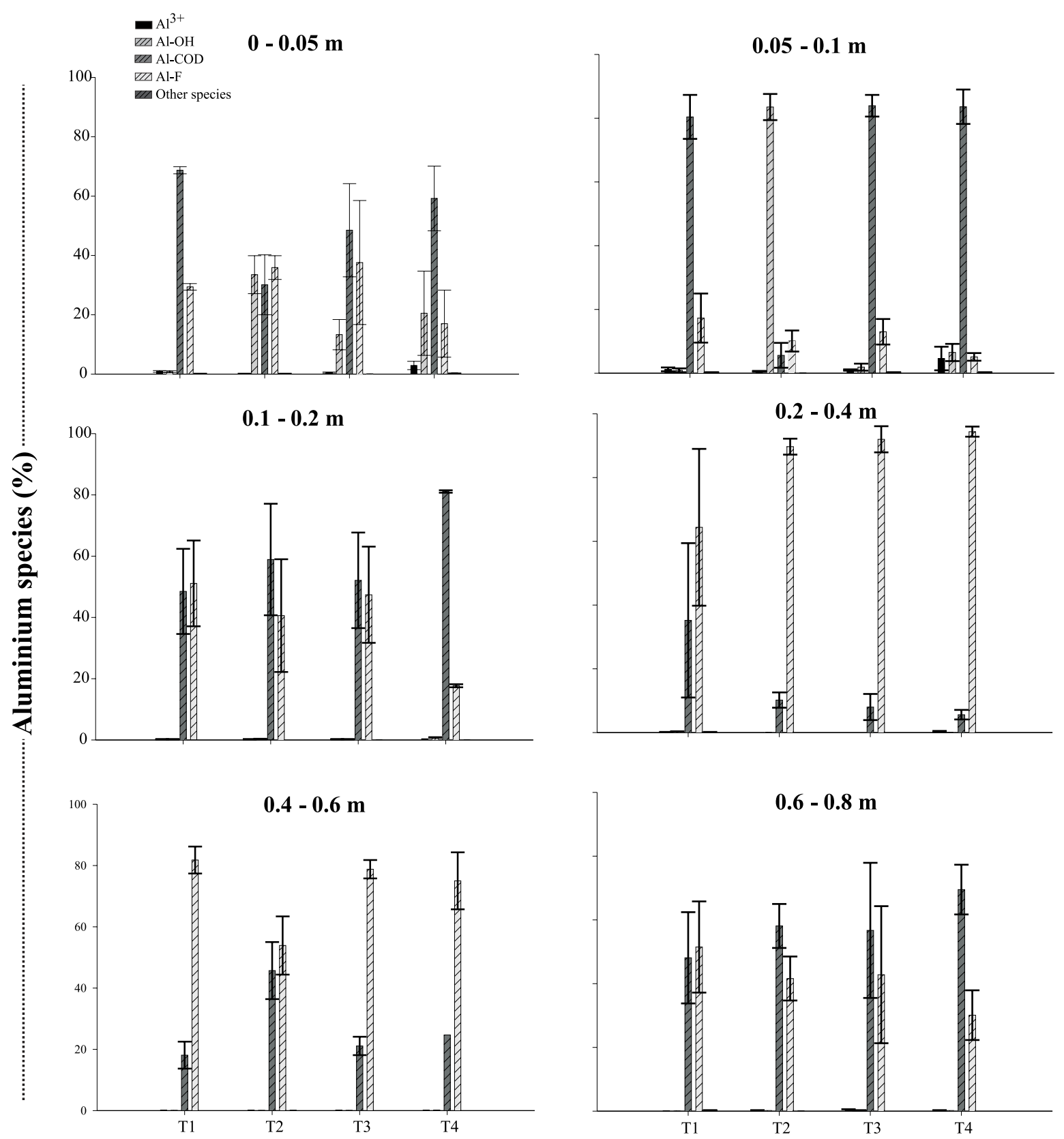

\section{Lime Treatment}

Figure 1 - Lime treatments, and chemical species of aluminum in the solution of an Oxisol under no-till system. T1 = control (without lime); $\mathrm{T} 2=4.5 \mathrm{t} \mathrm{ha}^{-1}$ of surface-applied lime (split in three annual applications of $1.5 \mathrm{t} \mathrm{ha}^{-1}$ ); $\mathrm{T} 3=4.5 \mathrm{tha}^{-1}$ of surface-applied lime (single application); $\mathrm{T} 4=4.5 \mathrm{t} \mathrm{ha}^{-1}$ of lime incorporated at $0.2 \mathrm{~m}$ depth. Vertical bars represent the mean deviation $(\mathrm{n}=3) . \mathrm{Al}-\mathrm{OH}=\mathrm{Al}(\mathrm{OH})^{2+}+\mathrm{Al}(\mathrm{OH})_{2}{ }^{+}+\mathrm{Al}(\mathrm{OH})_{3}{ }^{0} ; \mathrm{Al}-\mathrm{F}=\mathrm{Al}(\mathrm{F})^{2+}+\mathrm{Al}(\mathrm{F})_{2}{ }^{+}+\mathrm{Al}(\mathrm{F})_{3}{ }^{0}+\mathrm{Al}(\mathrm{F})_{4}{ }_{4}$. Other species: $\mathrm{AlHPO}_{4}^{+}$ $+\mathrm{Al}_{2} \mathrm{PO}_{4}^{3+}+\mathrm{AlSO}_{4}^{+}$.

with chloride and nitrate in this study are in accordance with Adams (1971), who stated that ionic pairs with these anions do not occur or are negligible, while sulfates can have a more expressive association. Nevertheless, the complexation of soluble Ca by DOC was not considered by Adams (1971) and other authors (Carvalho \& Raij, 1997; Nogueira \& Mozeto, 1990;
Chaves et al., 1991 e Csillag \& Kapoor, 1987). Such complexation plays an important role in soils where OM was accumulated due to soil management practices.

The complexation of calcium by organic anions has been related to a vertical mobilization of $\mathrm{Ca}$ in soils which received or accumulated surface organic residues, since these anions, after complexing 


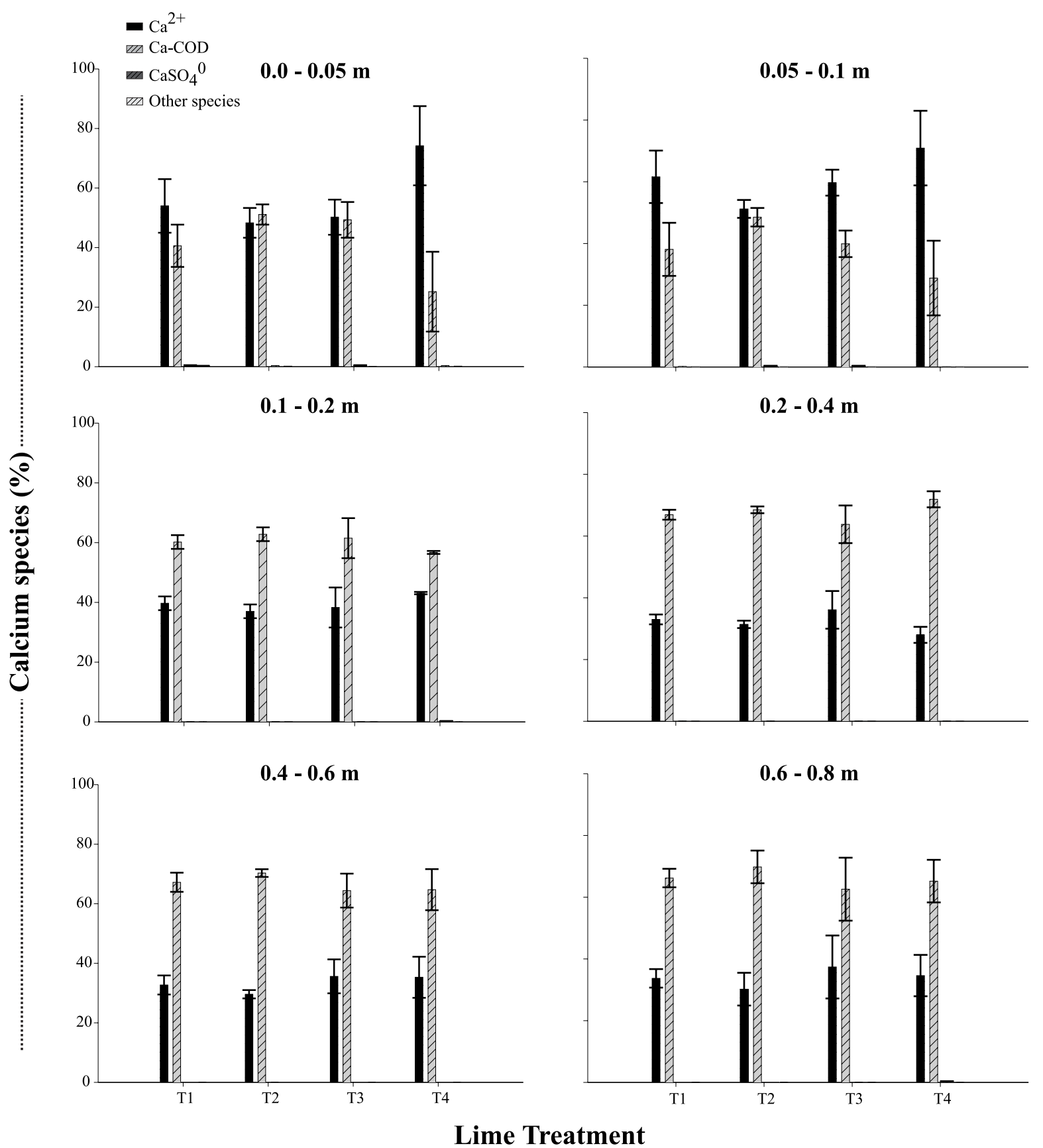

Figure 2 - Lime treatments, and chemical species of calcium in the solution of an Oxisol under no-till system. T1 = control (without lime); $\mathrm{T} 2=4.5 \mathrm{t} \mathrm{ha}^{-1}$ of surface-applied lime (split in three annual applications of $1.5 \mathrm{t} \mathrm{ha}^{-1}$ ); $\mathrm{T} 3=4.5 \mathrm{tha}^{-1}$ of surface-applied lime (single application); $\mathrm{T} 4=4.5 \mathrm{t} \mathrm{ha}^{-1}$ of lime incorporated at $0.2 \mathrm{~m}$ depth. Vertical bars represent the mean deviation $(\mathrm{n}=3)$. Other species: $\mathrm{CaCl}^{+} ; \mathrm{CaNO}_{3}^{+} ; \mathrm{CaF}^{+} ; \mathrm{CaHPO}_{4(\mathrm{aq})} ; \mathrm{CaH}_{2} \mathrm{PO}_{4}^{+}$.

the cation, cause a change in charges and favor leaching (Pavan, 1994; Ziglio et al., 1999; Franchini et al., 1999, 2001; Cassiolato et al., 2000). In the present study, there was high complexation of Ca by DOC down to the $0.8 \mathrm{~m}$ depth (Figure 2). So, there are indicators that this form of leaching does occur in areas under NT. Nevertheless, the ionic pairs between $\mathrm{Ca}$ and inorganic anions (sulfate, chloride, and nitrate) in the soil solution are also significant agents of cation mobilization along the soil profile (Soprano \& Alvarez, 1989; Pavan et al., 1984; Piirainem et al., 2002). The sulfate content in solution is directly related to the cation leaching potential (Piirainem et al., 2002). In the present study, the soluble sulfate content in the $0.1-0.2$ and $0.2-0.4 \mathrm{~m}$ layers was positively correlated to the calcium content in solution $(\mathrm{r}=0.60 ; P=0.04$ and $\mathrm{r}=0.62 ; P=0.03$, respectively), indicating that the $\mathrm{Ca}$ movement 
from the surface layers was, at least in part, related to sulfates.

DOC was the main ligand of $\mathrm{Mg}$ in the soil solution (Mg-DOC), but the free form $\mathrm{Mg}^{2+}$ was predominant (Figure 3). Among the inorganic anions, the interaction with sulfate $\left(\mathrm{MgSO}_{4}{ }^{0}\right)$ occurred in proportions close to $1 \%$. When performing ionic speciation in the solution of several soils with distinct chemical and physical characteristics in São Paulo State, Brazil, Nogueira \& Mozeto (1990) observed that the ionic pair $\mathrm{MgSO}_{4}{ }^{0}$ represented up to $1.5 \%$ of the total $\mathrm{Mg}$ species. Although on a smaller scale, there were interactions of magnesium with other inorganic anions, such as fluoride $\left(\mathrm{MgF}^{+}\right)$, phosphate $\left(\mathrm{MgHPO}_{4(\mathrm{aq})}\right)$, nitrate $\left(\mathrm{MgNO}_{3}^{+}\right)$, and chloride $\left(\mathrm{MgCl}^{+}\right)$, always under $0.5 \%$ of the total species of this cation, designated as

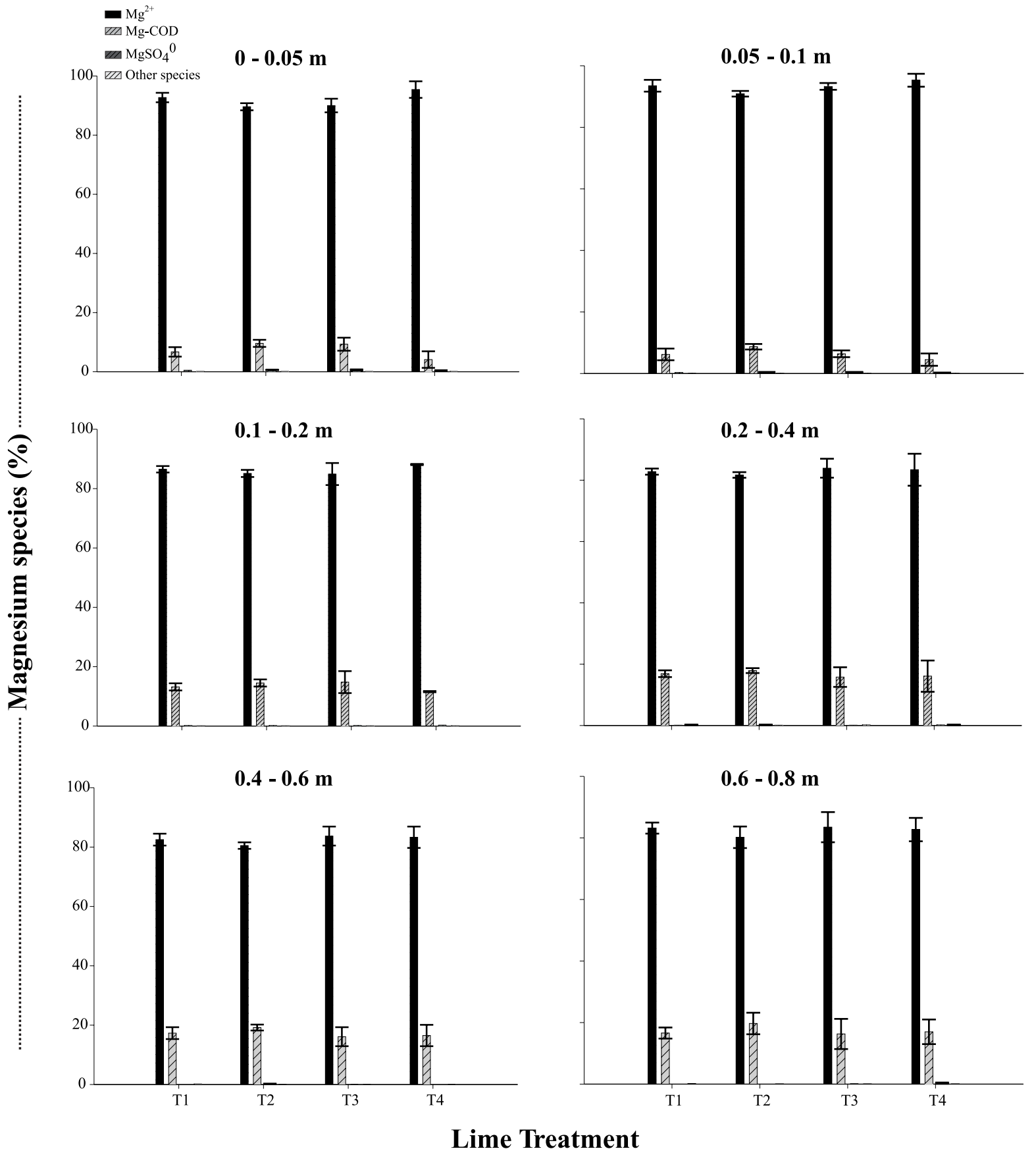

Figure 3 - Lime treatments, and chemical species of magnesium in the solution of an Oxisol under no-till system. T1 = control (without lime); $\mathrm{T} 2=4.5 \mathrm{t} \mathrm{ha}^{-1}$ of surface-applied lime (split in three annual applications of $1.5 \mathrm{t} \mathrm{ha}^{-1}$ ); $\mathrm{T} 3=4.5 \mathrm{t} \mathrm{ha}^{-1}$ of surface-applied lime (single application); $\mathrm{T} 4=4.5 \mathrm{tha}^{-1}$ of lime incorporated at $0.2 \mathrm{~m}$ depth. Vertical bars represent the mean deviation $(\mathrm{n}=$ 3). Other species: $\mathrm{MgCl}^{+} ; \mathrm{MgNO}_{3}^{+} ; \mathrm{MgF}^{+} ; \mathrm{MgHPO}_{4(\mathrm{aq})}$. 
"other species" in Figure 3. The magnesium complexes with chloride and nitrate have similar stability constants, but occupy insignificant proportions regarding the activities of these two anions in solution (Lindsay, 1979). Thus, just as with calcium, the contents of magnesium and sulfate in solution were positively correlated in the $0.1-0.2 \mathrm{~m}(\mathrm{r}=0.67 ; P=0.01)$ and $0.2-0.4$ $\mathrm{m}(\mathrm{r}=0.66 ; P=0.02)$ layers, which could be related to the joint leaching of these elements.
Potassium, unlike calcium and magnesium, was not complexed by organic anions in solution, being found mainly as a free ion $\left(\mathrm{K}^{+}\right)$. The percentage of ionic pairs for $\mathrm{K}\left(\mathrm{KCl}_{(\mathrm{aqu}}, \mathrm{KNO}_{3}\right.$, and $\left.\mathrm{KSO}_{4}^{-}\right)$represented a maximum of $0.2 \%$ of the total, regardless of the liming treatment or the sampling depth. In well drained soils, $\mathrm{K}$ complexes are usually of little relevance (Lindsay, 1979). However, the interaction of potassium with anions in the soil solution is important for leach-

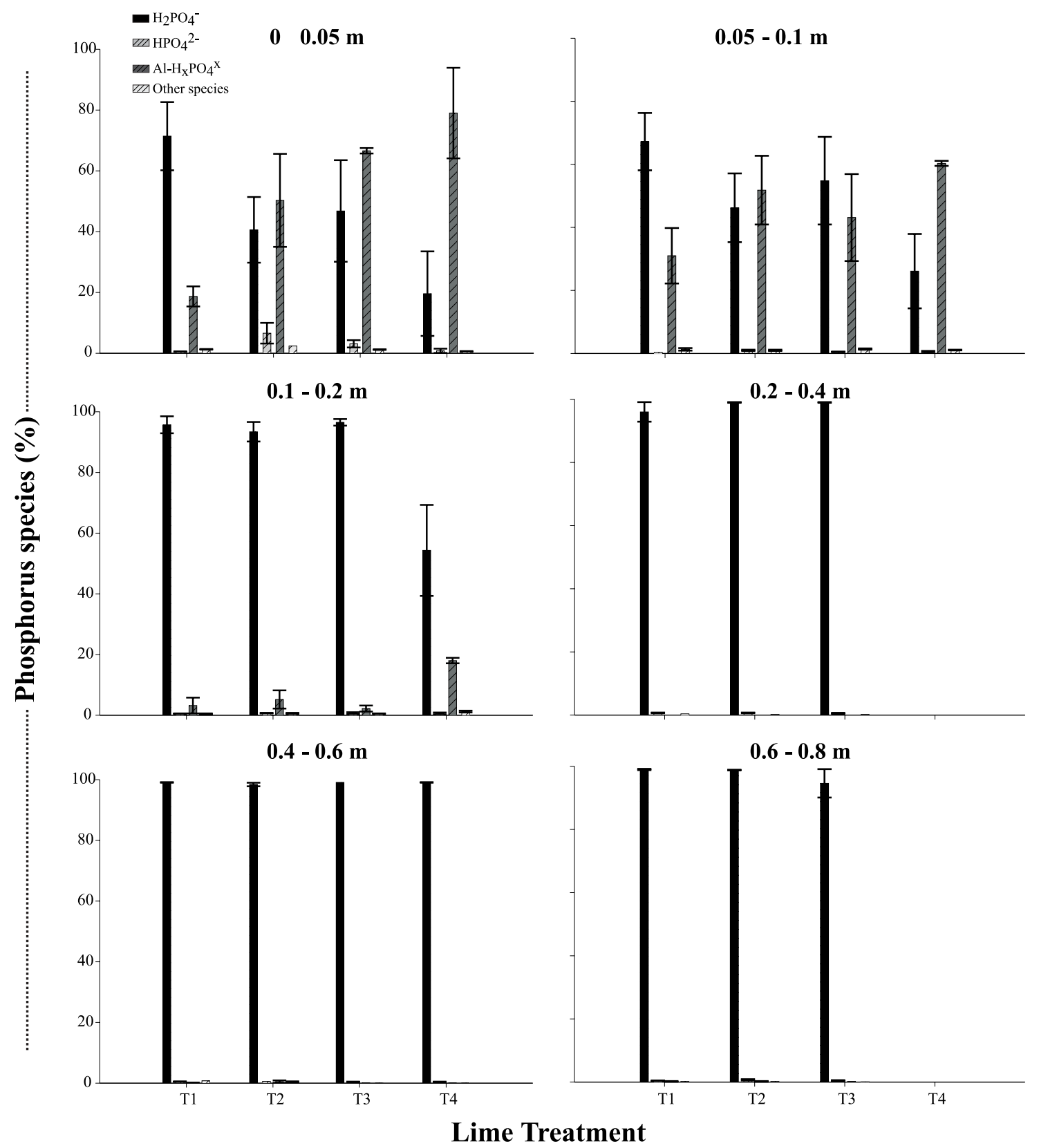

Figure 4 - Lime treatments, and chemical species of phosphorus in the solution of an Oxisol under no-till system. T1 = control (without lime); $\mathrm{T} 2=4.5 \mathrm{t} \mathrm{ha}^{-1}$ of surface-applied lime (split in three annual applications of $1.5 \mathrm{t} \mathrm{ha}^{-1}$ ); $\mathrm{T} 3=4.5 \mathrm{t} \mathrm{ha}^{-1}$ of surface-applied lime (single application); $\mathrm{T} 4=4.5 \mathrm{t} \mathrm{ha}^{-1}$ of lime incorporated at $0.2 \mathrm{~m}$ depth. Vertical bars represent the mean deviation $(\mathrm{n}=$ 3). $\mathrm{Al}-\mathrm{H}_{\mathrm{x}} \mathrm{PO}_{4}{ }^{\mathrm{x}}=\mathrm{AlHPO}_{4}^{+}+\mathrm{Al}_{2} \mathrm{PO}_{4}^{3+}$. Other species: $\mathrm{FeHPO}_{4}^{+} ; \mathrm{MnHPO}_{4}^{+} ; \mathrm{CaHPO}_{4(\mathrm{aq})} ; \mathrm{CaH}_{2} \mathrm{PO}_{4}^{+} ; \mathrm{MgHPO}_{4(\mathrm{aq})} ; \mathrm{KH}_{2} \mathrm{PO}_{4(\mathrm{aq})}$. 
ing - even though it is a monovalent cation, potassium needs accompanying anions to move along the soil profile (Rosolem et al., 2006). In this work, positive correlations were observed between potassium and chloride in the $0.1-0.2 \mathrm{~m}(\mathrm{r}=0.56 ; P=0.05)$ and $0.2-0.4 \mathrm{~m}(\mathrm{r}=0.51 ; P=0.05)$ layers, suggesting that a possible movement of potassium from the soil surface layers would be related to this anion. Potassium leaching, though, depends mainly on

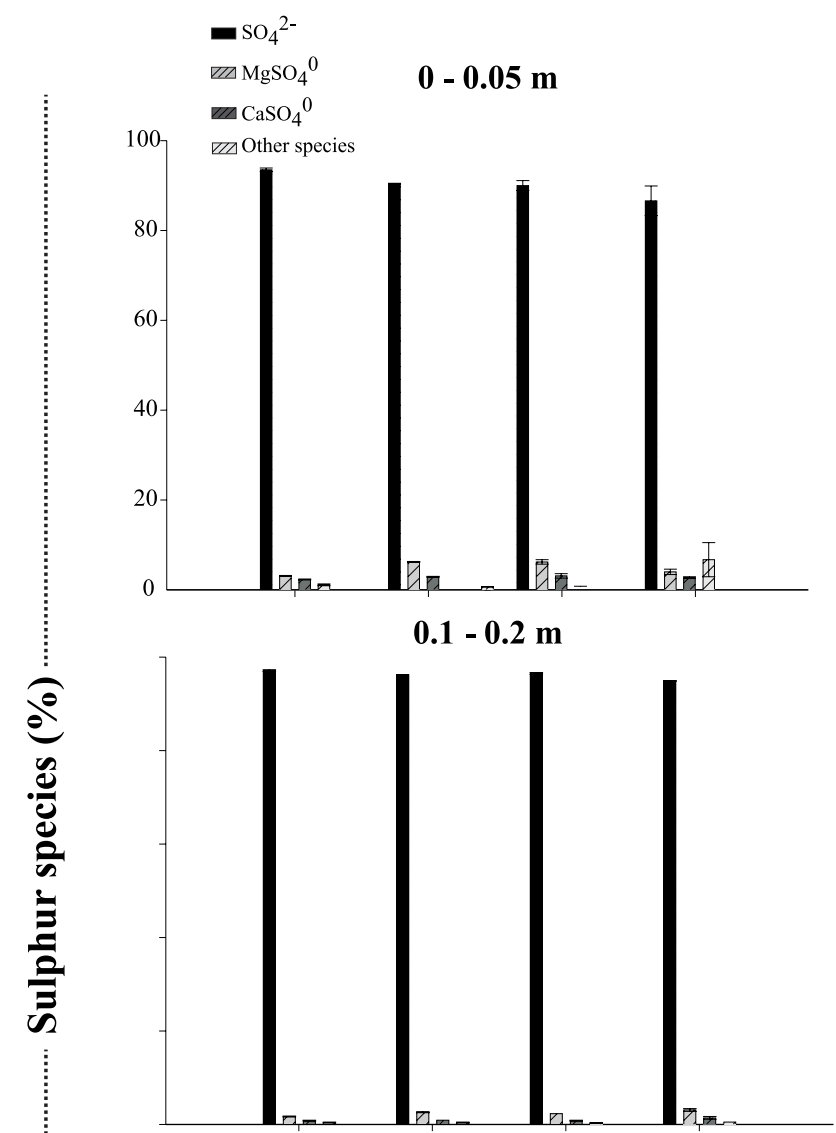

$0.4-0.6 \mathrm{~m}$

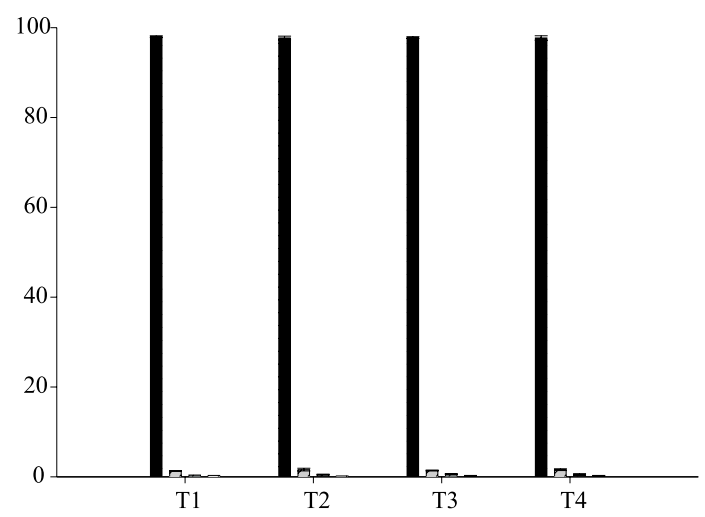

its content and on the water content in the soil profile (Rosolem et al., 2006).

The distribution of free forms of phosphorus $\left(\mathrm{H}_{2} \mathrm{PO}_{4}^{-}+\mathrm{HPO}_{4}^{2-}\right)$, which can be absorbed by plants, is dependent upon the soil solution $\mathrm{pH}$ values. There are approximately equal amounts of $\mathrm{H}_{2} \mathrm{PO}_{4}^{-}$and $\mathrm{HPO}_{4}^{2-}$ in a $\mathrm{pH}$ of 7.2 (Lindsay, 1979). In our study, the $\mathrm{pH}$ values of the soil solution were much smaller than 7.2 (Table 1), and thus, the $\mathrm{H}_{2} \mathrm{PO}_{4}^{-}$form was predominant
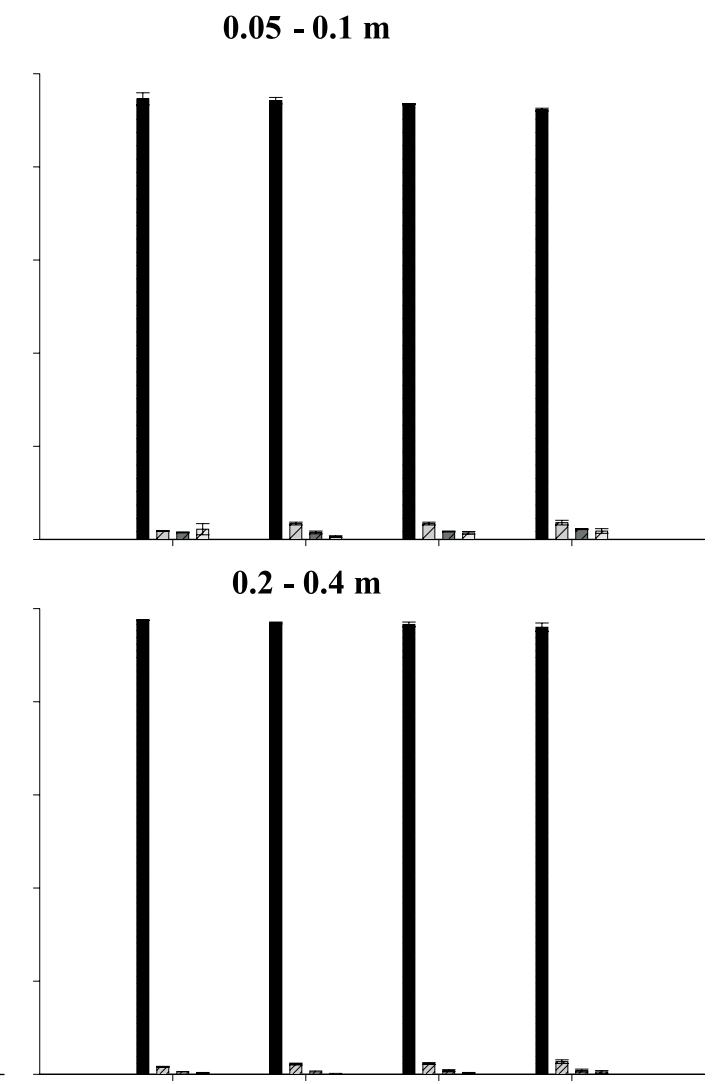

\section{Lime treatment}

Figure 5 - Lime treatments, and chemical species of sulphur in the solution of an Oxisol under no-till system. T1 = control (without lime); $\mathrm{T} 2=4.5 \mathrm{t} \mathrm{ha}^{-1}$ of surface-applied lime (split in three annual applications of $1.5 \mathrm{t} \mathrm{ha}^{-1}$ ); $\mathrm{T} 3=4.5 \mathrm{t} \mathrm{ha}^{-1}$ of surface-applied lime (single application); $\mathrm{T} 4=4.5 \mathrm{tha}^{-1}$ of lime incorporated at $0.2 \mathrm{~m}$ depth. Vertical bars represent the mean deviation $(\mathrm{n}=$ 3). Other species: $\mathrm{AlSO}_{4}^{+} ; \mathrm{NaSO}_{4}^{-} ; \mathrm{KSO}_{4}^{-}$. 
(Figure 4). There was also association of phosphorus with cations in the soil solution (ionic pair Al$\mathrm{H}_{\mathrm{x}} \mathrm{PO}_{4}{ }^{\mathrm{x}}+$ other species) (Figure 4), which can be explained by the fact that phosphate ions in the soil solution are strongly prone to interactions with $\mathrm{Al}, \mathrm{Fe}$, $\mathrm{Ca}$, and $\mathrm{Mg}$. The magnitude of bonding of phosphate with each of these cations depends on the soil type, with $\mathrm{Al}$ and $\mathrm{Fe}$ bonds more predominant in acid soils, and more $\mathrm{Ca}$ and $\mathrm{Mg}$ bonds in alkaline soils (Lindsay, 1979).

The proportion of $\mathrm{Al}-\mathrm{H}_{\mathrm{x}} \mathrm{PO}_{4}{ }^{\mathrm{x}}+$ other forms increased with liming, more markedly in the first two soil layers (Figure 4). Liming can reduce the efficiency of phosphorus fertilization in soils under NT due to the interaction between phosphate and $\mathrm{Ca}^{2+}$ (Machado \& Silva, 2001). It is suggested that liming could have caused an increase in the concentration of cations in solution $\left(\mathrm{Ca}^{2+}\right.$ and $\left.\mathrm{Mg}^{2+}\right)$, and the displacement of $\mathrm{Al}^{3+}$ from the soil exchange complex, increasing the formation of ionic pairs with phosphate. Nolla \& Anghinoni (2006) observed the presence of chemical species of phosphate with calcium and magnesium in the solution of an Oxisol under NT. Since the phosphate content is low in relation to cation content, the impact of reducing phosphate activity by bonding with cations is much larger than reducing cation activity by bonding with phosphates. Prochnow et al. (2006) pointed out a possible reduction of $\mathrm{P}$ availability to plants, due to the decrease in activity of phosphate ions by forming ionic pairs with cations in the soil solution.

Sulfur occurred in the soil solution, regardless of the liming treatments or sampling depth, as free sulfate ion $\left(\mathrm{SO}_{4}^{2-}\right)$ (Figure 5), the form it is absorbed by plant roots (Leustek \& Saito, 1999). The elements which formed more ionic pairs with sulfate were magnesium $\left(\mathrm{MgSO}_{4}{ }^{0}\right)$ and calcium $\left(\mathrm{CaSO}_{4}{ }^{0}\right)$. In smaller proportions, there were associations with aluminum $\left(\mathrm{AlSO}_{4}^{+}\right)$, sodium $\left(\mathrm{NaSO}_{4}^{-}\right)$and potassium $\left(\mathrm{KSO}_{4}^{-}\right)$, designated as "other species" (Figure 5). On average, in the first two soil layers, the proportion occupied by the free $\mathrm{SO}_{4}^{2-}$ ion varied from 90 to $93 \%$, while the values reached $98 \%$ in the other layers, indicating that high cation concentrations tend to reduce the presence of sulfur as $\mathrm{SO}_{4}^{2-}$ in the soil solution.

Nitrate occurred predominantly in the free form $\left(\mathrm{NO}_{3}{ }^{-}\right.$) (average of $99.8 \%$ ) in the soil solution, regardless of the liming treatment or sampling depth, indicating little interaction with cations in solution. Performing a chemical speciation in a percolated solution, Amaral et al. (1998) reported that the nitrate ion was found only in the free form. Chloride also occurred mainly as free ions $\left(\mathrm{Cl}^{-}\right)$(average of 99.9\%), without liming treatment or sampling depth effects.

\section{CONCLUSIONS}

Aluminum forms complex mainly with dissolved organic carbon and fluoride in the soil solution under the no-till system. The complexation of aluminum by dissolved organic carbon is not influenced by soil acidity amelioration by liming.

Surface or incorporated application of lime does not affect $\mathrm{Ca}$ and $\mathrm{Mg}$ speciation in the soil profile solution. Dissolved organic carbon is the main complexing agent with both cations in the soil solution under the no-till system.

The proportion occupied by the free forms of phosphorus $\left(\mathrm{H}_{2} \mathrm{PO}_{4}^{-}+\mathrm{HPO}_{4}^{2-}\right)$ in the soil solution was influenced by liming due to the increment in cationassociated forms, mainly in the soil surface layers.

\section{REFERENCES}

ADAMS, F. Ionic concentrations and activities in soil solutions. Soil Science Society of America Proceedings, v.35, p.420426, 1971.

ADAMS, M.L.; McINTOSH, P.D.; PATTERSON, R.D.; POWELL, K.J. Aluminium speciation in seasonally dry high country soils, South Island, New Zealand. Australian Journal of Soil Research, v.37, p.1005-1015, 1999.

ALlEONI, L.R.F.; ZAMBROSI, F.C.B.; MOREIRA, S.G.; PROCHNOW, L.I.; PAULETTI, V. Liming and electrochemical attributes of an Oxisol under no tillage. Scientia Agricola, v.60, p.119-123, 2003.

ALlEONI, L.R.F.; CAMBRI, M.A.; CAIRES, E.F. Atributos químicos de um Latossolo de cerrado sob plantio direto, de acordo com doses e formas de aplicação de calcário. Revista Brasileira de Ciência do Solo, v.29, p.923-934, 2005.

ÁlVAREZ, E.; MONTERROSO, C.; FERNÁNDES MARCOS, M.L. Aluminium fractionation in Galicia (NW Spain) forest soils as related to vegetation and parent material. Forest Ecology and Management, v.166, p.193-206, 2002.

AMARAL, F.C.S.; VETTORAZZO, S.C.; CHITOLINA, J.C. Efeito do fluoreto de cálcio e do carbonato de cálcio na composição química da solução percolada de um solo ácido. Revista Brasileira de Ciência do Solo, v.22, p.379-385, 1998.

AMARAL, S.F.; ANGHINONI, I. Alteração de parâmetros químicos do solo pela reaplicação superficial de calcário no sistema plantio direto. Pesquisa Agropecuária Brasileira, v.36, p.695702, 2001.

BERTOL, I.; GUADAGNIN, J.C.; GONZALEZ, A.P.; AMARAL, A.J.; BRIGNONI, L.F. Soil tillage, water erosion, and calcium, magnesium and organic carbon losses. Scientia Agricola, v.62, p.578-584, 2005.

CAIRES, E.F.; CHUERI, W.A.; MADRUGA. E.F.; FIGUEIREDO, A. Alterações de características químicas do solo e resposta da soja ao calcário e gesso aplicados na superfície em sistema de cultivo sem preparo do solo. Revista Brasileira de Ciência do Solo, v.22, p.27-34, 1998.

CAIRES, E.F.; FONSECA, A.F.; MENDES, J.; CHUEIRI, W.A.; MADRUGA, E.F. Produção de milho, trigo e soja em função das alterações das características químicas do solo pela aplicação de calcário e gesso na superfície, em sistema de plantio direto. Revista Brasileira de Ciência do Solo, v.23, p.315-327, 1999.

CAIRES, E.F.; BANZATO, D.A.; FONSECA, A.F. Calagem na superfície em sistema de plantio direto. Revista Brasileira de Ciência do Solo, v.24, p.161-169, 2000. 
CAIRES, E.F.; KUSMAN, M.T.; BARTH, G.; GARBUIO, F.J.; PADILHA, J.M. Alterações químicas do solo e resposta do milho à calagem e aplicação de gesso. Revista Brasileira de Ciência do Solo, v.28, p.125-136, 2004.

CAIRES, E.F.; ALLEONI, L.R.F.; CAMBRI, M.A.; BARTH, G. Surface application of lime for crop grain production under a no-till system. Agronomy Journal, v.97, p.791-798, 2005.

CAIRES, E.F.; BARTH, G.; GARBUIO, F.J. Lime application in the establishment of a no-till system for grain crop production in Southern Brazil. Soil Tillage Research, v.89, p.3-12, 2006 a.

CAIRES, E.F.; CHURKA, S.; GARBUIO, F.J.; FERRARI, R.A.; MORGANO, M.A. Soybean yield and quality a function of lime and gypsum applications. Scientia Agricola, v.63, p.370-379, 2006b.

CAMBRI, M.A.; ALLEONI, L.R.F.; CAIRES, E.F. Dissolved organic carbon and aluminum speciation in an Oxisol under no-tillage. In: INTERNATIONAL MEETING OF THE INTERNATIONAL HUMIC SUBSTANCES SOCIETY, 12., São Pedro, 2004. Proceedings. São Pedro: Embrapa, 2004. p.689690.

CARVALHO, M.C.S.; RAIJ, B. van. Calcium sulphate, phosphogypsum and calcium carbonate in the amelioration of acid subsoils for root growth. Plant and Soil, v.192, p.37-48, 1997.

CASSIOLATO, M.E.; MEDA, A.R.; PAVAN, M.A.; MIYAZAWA, M.; OLIVEIRA, J.C. Evaluation of oat extracts on the efficiency of lime in soil. Brazilian Archives of Biology and Technology, v.43, p.533-536, 2000.

CHAVES, J.C.D.; PAVAN, M.A.; MIYAZAWA, M. Especiação química da solução do solo para interpretação da absorção de cálcio e alumínio por raízes de cafeeiro. Pesquisa Agropecuária Brasileira, v.26, p447-453, 1991.

CIOTTA, M.N.; BAYER, C.; ERNANI, P.R.; FONTOURA, S.M.V.; WOBETO, C. ALBUQUERQUE, J.A. Manejo da calagem e os componentes da acidez de Latossolo Bruno em plantio direto. Revista Brasileira de Ciência do Solo, v.28, p.317-326, 2004.

CSILLAG, J.; KAPOOR, B.S. The importance of the study of chemical speciation in natural waters and soil solutions. Clay Research, v.6, p.87-96, 1987.

DRABECK, O.; MLADKOBA, L.; BORUVKA, L.; SZAKOVA, J.; NIKODEM, A.; NEMECEK, K. Comparison of water-soluble and exchangeable forms of $\mathrm{Al}$ in acid forest soils. Journal of Inorganic Biochemistry, v.99, p.1788-1795, 2005.

EMPRESA BRASILEIRA DE PESQUISA AGROPECUÁRIA EMBRAPA. Manual de análises químicas de solos, plantas e fertilizantes. 2. ed. Rio de Janeiro, Embrapa-CNPS, 1997. 212 p.

FONTES, M.P.F.; ALLEONI, L.R.F. Electrochemical attributes and availability of nutrients, toxic elements and heavy metals in tropicals soils. Scientia Agricola, v.63, p.589-608, 2006.

FRANCHINI, J.C.; MIYAZAWA, M.; PAVAN, M.A.; MALAVOLTA, E. Dinâmica de íons em solos ácidos lixiviado com extratos de resíduos de adubos verdes e soluções puras de ácidos orgânicos. Pesquisa Agropecuária Brasileira, v.34, p.2267-2276, 1999

FRANCHINI, J.C.; MEDA, A.R.; CASSIOLATO, M.E.; MIYAZAWA, M.; PAVAN, M.A. Potencial de extratos de resíduos vegetais na mobilização de calcário no solo por método biológico. Scientia Agricola, v.58, p.357-360, 2001.

GUSTAFSSON, J.P. Visual MINTEQ. Available at: http:// www.lwr.kth.se/English/OurSoftware/Vmintes. Accessed 26 Oct. 2004

HUE, N.V.; CRADDOCK, G.K.; ADAMS, F. Effect of organic acids on aluminium toxicity in subsoils. Soil Science Society of America Journal, v.50, p.28-34, 1986

KAMINSKI, J.; SANTOS, D.R.; GATIBONI, L.C.; BRUNETO, G.; SILVA, L.S. Eficiência da calagem superficial e incorporada precedendo o sistema plantio direto em um argissolo sob pastagem natural. Revista Brasileira de Ciência do Solo, v.29, p.573580,2005
KRISHNAMURTIAND, G.S.R.; NAIDU, R. Solid-solution speciation and phytoavailability of copper and zinc in soil. Environmental Science of Technology, v.36, p.2645-2651, 2002.

LEUSTEK, T.; SAITO, K. Sulfate transport and assimilation in plants. Plant Physiology, v.120, p.637-643, 1999

LINDSAY, W.L. Chemical equilibria in soils. New York: John Wiley, 1979. 450p.

LINDSAY, W.L.; WALTHALL, P.M. The solubility of aluminum in soils. In: SPOSITO, G. (Ed) The environmental chemistry of aluminum. 2.ed. Boca Raton: Lewis Publishers, 1996. p. 333-361.

MACHADO, P.L.O.A.; SILVA, C.A. Soil management under notillage systems in the tropics with special reference to Brazil. Nutrient Cycling in Agroecosystems, v.61, p.119-130, 2001.

MERINO, A.; MACÍAS, F.; GARCÍA-RODEJA, E. Aluminum dynamics in experimental acidified soils from a humid-temperate region of south Europe. Chemosphere, v.36, p.1137-1142, 1998.

NEMETH, K.; MENGEL, K.; GRIMME, H. The concentration of $\mathrm{K}, \mathrm{Ca}$ and $\mathrm{Mg}$ in the saturation extract in relation to exchangeable K, Ca, Mg. Soil Science, v.109, p.179-185, 1970.

NOGUEIRA, A.R.A.; MOZETO. A.A. Interações químicas do sulfato e do carbonato de cálcio em seis solos paulistas sob vegetação de cerrado. Revista Brasileira de Ciência do Solo, v.14, p.1-6, 1990.

NOLLA, A.; ANGHINONI, I. Atividade e especiação química na solução afetadas pela adição de fósforo em Latossolo sob plantio direto em diferentes condições de acidez. Revista Brasileira de Ciência do Solo, v.30, p.955-963, 2006.

PAVAN, M.A.; BINGHAN, F.T.; PRATT, P.F. Redistribution of exchangeable calcium, magnesium, and aluminum following lime or gypsum applications to a Brazilian Oxisol. Soil Science Society of America Journal, v.48, p.33-38, 1984.

PAVAN, M.A.; BLOCH, M.F.; ZEMPULSKI, H.C.; MIYAZAWA, M.; ZOCOLER, D.C. Manual de análise química do solo e controle de qualidade. Londrina: Instituto Agronômico do Paraná, 1992. 38 p. (Circular, 76).

PAVAN, M.A. Movimentação de calcário no solo através de técnicas de manejo da cobertura vegetal em pomares de macieira. Revista Brasileira de Fruticultura, v.16, p.86-91, 1994.

PIIRAINEM, S.; FINÉR, L.; STARR, M. Deposition and leaching of sulphate and base cations in mixed boreal forest in eastern Finland. Water, Air and Soil Pollution, v.131, p.185-204, 2002.

PROCHNOW, L.I.; BIASIOLI, G.M.; RODELLA, A.A.; JESUS, S.L.; MONTES, C.R.; ALLEONI, L.R.F. Activity of phosphorus of synthetic Fe-K-P compounds in superphosphate fertilizers as affected by $\mathrm{pH}$ and ionic strength. Revista Brasileira de Ciência do Solo, v.30, p.467-474, 2006.

RHEINHEIMER, D. S.; SANTOS, E.J.S.; KAMINSKI, J.; XAVIER, F.M. Aplicação superficial de calcário no sistema plantio direto consolidado em solo arenoso. Ciência Rural, v.30, p.263268, 2000.

ROSOLEM, C.A.; GARCIA, R.A.; FOLONI, J.S.S.; CALONEGO, J.C. Lixiviação de potássio no solo de acordo com suas doses aplicadas sobre palha de milheto. Revista Brasileira de Ciência do Solo, v.30, p.813-819, 2006.

SILVEIRA, M.L.A. Dissolved organic carbon and bioavailability of $\mathrm{N}$ and $\mathrm{P}$ as indicators of soil quality. Scientia Agricola, v.62, p.502-508, 2005.

SOPRANO, E.; ALVAREZ, V.H. Nutrientes lixiviados de colunas de solo tratados com diferentes sais de cálcio. Revista Brasileira de Ciência do Solo, v.13, p.25-29, 1989.

SPOSITO, G. Chemical equilibrium and kinetics in soils. New York: Oxford Univesity Press, 1994. 288 p

TACK, F.M.; VERLOO, M.G. Chemical speciation and fractionation in soil and sediment heavy metal analysis: a review. International Journal of Environmental Analytical Chemistry, v.59, p.225-238, 1995 
VANCE, G.F.; STEVENSON, F.J.; SIKORA, F.J. Environmental chemistry of aluminum-organic complexes. In: SPOSITO, G. (Ed) The environmental chemistry of aluminum. 2.ed. Boca Raton: Lewis Publishers, 1996. p.169-220.

WOLT, J.D. Obtaining soil solution: laboratory methods. In: WOLT, J.D. Soil solution chemistry: applications to environmental science and agriculture. New York: John Wiley, 1994. p.95120

ZAMBROSI, F.C.B.; ALLEONI, L.R.F.; CAIRES, E.F. Nutrient concentration in soil water extracts and soybean nutrition in response to lime and gypsum applications to an acid Oxisol under no-till system. Nutrient Cycling in Agroecosystems, v.79, p.169-179, 2007.
ZIGLIO, C.M.; MIYAZAWA, M.; PAVAN, M.A. Formas orgânicas e inorgânicas de mobilização do cálcio no solo. Brazilian Archives of Biology and Technology, v.42, p.257-262, 1999.

Received January 02, 2007

Accepted October 15, 2007 